Nutrition and HIV

1 Correlation between the immuno-virological response and the nutritional profile of treatment-experienced HIV-infected patients in the East region of

\title{
Cameroon
}

Abba Aissatou ${ }^{1,2,}{ }^{*}$, Joseph Fokam ${ }^{1,2,3,}{ }^{*}$, Rachel Simo Kamgaing ${ }^{1}$, Junie Flore Yimga ${ }^{1}$, Aude Christelle Ka'e $^{3}$, Alex Durand Nka ${ }^{1}$, Michel Carlos Tommo Tchouaket ${ }^{1,2}$, Ambe Collins Chenwi ${ }^{1}$, Ezechiel Ngoufack Jagni Semengue ${ }^{1}$, Alexis Ndjolo ${ }^{1,3}$, Samuel Martin Sosso ${ }^{1}$

7

1. Chantal BIYA International Reference Centre for research on HIVIAIDS prevention and management (CIRCB), Yaoundé, Cameroon;

2. School of Health Sciences, Catholic University of Central Africa, Yaoundé, Cameroon;

3. Faculty of Medicine and Biomedical Sciences (FMBS), University of Yaoundé I, Yaoundé, Cameroon.

*Corresponding authors:

Abba Aissatou and Joseph Fokam, Emails: aichabba@ymail.com; fokamjoseph@circb.cm 


\section{Nutrition and HIV}

\section{Abstract}

31 Background: HIV management remains concerning and even more challenging in the frame of comorbidities like malnutrition that favors disease progression and mortality in resource-limited settings (RLS).

Objective: To evaluate the correlation between immuno-virological responses and the nutritional profile of HIV-infected individuals receiving antiretroviral therapy (ART).

Methods: A cross-sectional study was conducted from October to December 2018 among 146 consenting participants enrolled in two health facilities of the East-Region of Cameroon. Socio-demographic data, basic clinical information and treatment history were collected; blood samples were collected by venipuncture for laboratory analysis (HIV-1 viral load, CD4-CD8 Tcells measurement and biochemical analysis) performed at the "Chantal Biya" International Reference Center", Yaounde, Cameroon. Nutritional profile was evaluated using anthropometric and biochemical parameters. Data were analyzed using Excel 2016, Graph pad prism version 6 and R.version3.5.0; Spearman correlation was used; with $p<0.05$ considered statistically significant.

Results: Median [IQR] age was 42 [33-51] years, 76.0\% ( 111/146) were female and median [IQR] duration on ART was 54 [28-86] months. Of these participants, 11.6\% (17/146) were underweight based on the body mass index and $4.7 \%$ (7/146) were at the stage of advanced weight loss. According to immunovirological responses, 44.5\% (65/146) were immunocompromised (CD4<500 cell/ $\mu \mathrm{l})$ and $75.3 \%$ (110/146) had an undetectable viremia (<40 copies/mL). CD4 count inversely correlated with total protein concentration $(r=-0.18, p=0.030)$ and viremia was inversely correlated with total cholesterol $(r=-0.65$; $p=0.001)$, and positively correlated with total protein $(r=0.28 ; p<0.001)$ and seemingly with triglycerides $(r=0.27 ; p=0.070)$ concentrations.

Conclusion: In this RLS with patients having about five years of ART-experience, half are immunocompromised while the majority have achieved good virological response. Interestingly, one out of eight patients might be experiencing malnutrition. Specifically, increasing CD4 may favour hypoproteinemia while increasing viral load may prone hyper-proteinemia and hypo-cholesterolemia. Further studies are needed in RLS with high burden of HIV-infection.

Key words: Nutritional profile, HIV, ART, CD4, Viral load, East-Cameroon. 


\section{Introduction}

The human immunodeficiency virus (HIV) targets the immune system and weakens the surveillance and defense system of the body against infections, leading to susceptibility of HIV infected individuals to a wide range of infections normally cleared by the immune system of a healthy/immunocompetent individual [1]. HIV can therefore cause several health complications including opportunistic infections, oxidative stress, wasting syndrome, as well as malnutrition [2].

Malnutrition is one of the major complications of HIV infection [3] and has been recognized under the banner of 'wasting syndrome' as a significant prognostic factor of advanced disease [4]. Of note, malnutrition is defined as unhealthy diets deficient in micronutrients and micronutrient imbalances, which can disrupt the function of various immune system [5]. Specifically, under-nutrition impairs the immune system mechanism and thus impairs the host response against micro-organisms. The consequence of this impairment is an increase in both incidence and severity of infections [6]. Particularly, HIV infection and insufficient nutritional intake are part of a vicious cycle that contributes to immunodeficiency and poor health outcomes [7].

HIVIAIDS and malnutrition have a synergistic interaction within the host. In effect, malnutrition increases the risk of HIV pathogenesis, while HIV in turn triggers malnutrition by depleting the immune system from nutrient intake, absorption and utilization [8]. There is a complex triangulation mechanism between malnutrition, the immune system and HIV infection, in which malnutrition elicits immune system dysfunctions which in turn promotes increased vulnerability of the host to infection, while the latter intensifies the severity of malnutrition [9].

Progress in scaling-up HIV treatment (23.3 million, representing 62\% global coverage) have reduced associated mortality and morbidity, thus making HIV a chronic infection, even in sub-Saharan African countries where $70 \%$ of the global epidemics is concentrated [10-13]. Thus, for an enhanced performance of ART programs, it is postulated that nutritional interventions should become an integral component in the management of people living with HIV (PLHIV). Such strategy suggests that improved attention to diet and nutrition could normalize protein profiling, fatty acids, copper and iron, which in turn would harness the immune function and subsequently optimize ART acceptability, adherence and effectiveness [14,15]. In spite of the declining burden of HIV at country-level (5.4\% in 2004 to $2.7 \%$ in 2019), Cameroon is still experiencing a generalized epidemiology [16-18]. The national strategy for the fight against HIVIAIDS in Cameroon recommends a nutritional supplement to subside the current $14.1 \%$ persistent rate of malnutrition for PLHIV, and in turn to support ART response $[19,20]$. Interestingly, the East region of Cameroon has a very high burden of HIV infection (5.6\%), 43.01\% patients are on ART [18,21], while up 
91 to $13.7 \%$ of these patients are known to be undernourished [20]. Thus, these high rates of both HIV

92 infection and malnutrition call for further investigations to improve the management of this comorbidity in similar settings of sub-Saharan Africa.

We therefore sought to study the correlation between the immuno-virological response and the nutritional profile among ART-experienced HIV-infected patients in the East-region of Cameroon.

\section{Materials and methods}

\section{Study design, sampling method and eligibility criteria}

A cross-sectional and analytical study was carried-out among PLHIV receiving ART at two heath facilities in the East region of Cameroon, Bertoua Regional Hospital (BRH); and Nkolbikon Catholic Health Center (NCHC), during three months (from October through December 2018).

Following a consecutive sampling, the required minimum sample size for the study was calculated based on the prevalence of undernourished PLHIV in the East-region of Cameroon (13.7\%) [20], as per the following statistical formula:

$\mathrm{N}=\frac{\mathrm{Za}^{2} \times \mathrm{P}(1-\mathrm{P})}{\mathrm{d}^{2}}$

With "Z" equal to 1.96 at $95 \%$ confidence interval, with "P" the prevalence of undernourished PLHIV in our study setting (13.7\%) [20], and "d" being the error rate set at 6\% (0.06); after numerical application, we obtained a minimum sample size, "N" = 126.2, rounded-up to a minimum of 127 participants to be enrolled in the study.

110 Eligibility criteria were every individual: 1) with confirmed HIV-positive; 2) on ART for at least six (06) 111 months; 3) registered in one of the study sites; and 4) aged 15 years and above. Following these criteria,

112 a total of 146 participants were enrolled.

\section{Phlebotomy and sample shipment}

114 A standard questionnaire was administered to each participant by interviewers trained on the study 115 protocol. Blood sample was then collected (in dry and Ethylenediaminetetraacetic acid test tubes of $4 \mathrm{ml}$ ) 116 by venipuncture with the help of trained phlebotomists. After collection, blood samples were transferred 117 from the sampling sites to the BRH laboratory for packaging and shipment. Only dry test tubes were 118 centrifuged and separated in aliquots for biochemical analysis. After centrifugation, racks of all samples 119 were placed in ice packed isothermal bags, and samples were then sent for laboratory analysis to the 
120 study reference institution (Chantal BIYA International Reference Center for Research on HIVIAIDS

121 prevention and management), located in Yaoundé, the capital city of Cameroon.

\section{Clinical and laboratory procedures}

123 During routine clinic attendance at the study sites, the standard questionnaire (S1 file) administered to all 124 participants enabled us to retrieve information covering socio-demographic data, treatment history and 125 food habits, as well as basic clinical information and all biological parameters performed.

126 CD4 and CD8 cell count were performed using the Cyflow Counter-Sysmex Partec as per the 127 manufacturer's instructions with results reported as the number of positive cells per microliter of blood 128 (http://www.nsplucknow.com/pdf/CyFlow_Counter_Bro_EN.pdf). CD4 results were then interpreted as 129 follows: no immunodeficiency (above or equal to 500); moderate immunodeficiency (between 350 and 130 499); advanced immunodeficiency (between 200 and 349) and severe immunodeficiency (below 200) 131 [22]. Regarding the interpretation of CD8 cell results: no immunodeficiency was defined as absolute value 132 above 220 cells while immunodeficiency was below 220, as previously described [23]. Viral load 133 measurement was performed using the Abbott m2000RT Real Time PCR as-per the manufacturer's 134 instructions (Abbott Laboratories, USA), with a lower detection threshold of $40 \mathrm{HIV}-1 \mathrm{RNA}$ copies/mL and 135 an upper detection threshold of $10,000,000$ copies $/ \mathrm{mL}$

136 (www.abbottmolecular.com/products/infectious-diseases/realtime-pcr/hiv-1-assay).

137 The nutritional profile of participants was evaluated based on biochemical parameters: albumin, calcium, 138 glucose, iron, magnesium, total cholesterol, triglycerides, total protein and anthropometric parameters of 139 which the body mass index (BMI), nutritional risk index (NRI) and weight loss percentage (WLP). 140 Biochemical analysis was performed using BT-3000 Plus as per manufacturer's instructions 141 (https://www.chema.com/chema/automation_it_files/Biotecnica\%20BT3000.pdf). BMI was defined as an 142 indicator of chronic energy malnutrition and was calculated by dividing the weight (in $\mathrm{Kg}$ ) by the height 143 squared (in square meter):

$144 \quad B M I=\left[\frac{\text { Weight }}{\text { Height }^{2}}\right] ;[24]$.

145 The NRI, a nutritional status assessment index recommended in national nutritional programs, was 146 estimated by the following formula:

$147 N R I=\left(1.519 \times\right.$ serum albumin,,$\left.\frac{\mathrm{g}}{\mathrm{dL}}\right)+\frac{41.7 \times \text { present weight }(\mathrm{kg})}{\text { ideal body weight }(\mathrm{kg})} ;[25]$.

148 WLP is used to assess the risk of malnutrition and determined by the formula 
$W L P=\left(\frac{\text { Weight Lost }}{\text { Usual Weight }}\right) \times 100$

Weight Lost $=$ Usual Weight - Present weight; [26].

\section{Statistical Analysis}

153 Collected data were entered on Microsoft Excel 2016 sheets and analyzed using the software GraphPad

154 Prism version 6.0. Dependent variables were immune status and viremia, while independent variables were anthropometric and biochemical parameters. Correlation analysis were performed using Spearman correlation test; with $p<0.05$ considered statistically significant. The software $R$ version 3.5.0. was used for backward multiple regression with AIC (Akaike Information Criteria) selection. P-values $\leq 0.2$, obtained in bivariate analysis, were considered for the multivariate analysis.

\section{Ethical Considerations}

160 The study was conducted in compliance with the core principles of the Helsinki declaration: an 161 administrative authorization was issued; ethical clearance was obtained from the National Ethics

162 Committee for Research on Human Health (ref N0 2018/06/1055/CE/CNERSH/SP); written informed consent were obtained from all the participants; data were processed using unique identifiers to ensure confidentiality; laboratory results were returned to participants for possible benefits in their clinical management; and counseling on good nutritional habits and healthy lifestyles was provided to all.

\section{Results}

\section{Socio-demographic and clinical characteristics of the population}

Of the total of 146 participants enrolled, 88.4\% (129) at Bertoua regional hospital (BRH) and $11.6 \%$ (17) at Nkolbikon Catholic Health Center (NCHC). Among them, 76.0\%(111) were females giving a sex ratio (female/male) of 3 . The median age was 42 [IQR: 33-51] years (Table 1).

172 The median duration on ART was 54 [IQR: 28-86] months. All participants were on first-line ART regimen

173 consisting of two nucleoside reverse transcriptase inhibitors (NRTIs) and one non-NRTI (NNRTI).

\section{Nutritional profile}

176 Of the 146 participants, BMI results revealed that only a proportion of $11.6 \%$ (17) was undernourished.

177 According to NRI, 91.1\% (133) were normal, while referring to WLP, 60.9\% (89) had a normal weight. 
178 Only $2.7 \%$ (4) of participants suffered from hypoalbuminemia, 19.9\% (29) from hyper-proteinemia and

179 20.5\%(30) from hypo-cholesterolemia (Table 2).

\section{Immuno-virological status}

181 The median [IQR] CD4+ T cell lymphocyte count was 547 [385-759] cells/ul, and 44.5\% (65) were

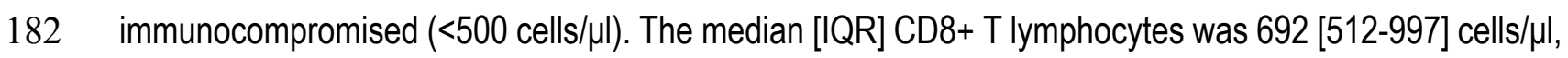

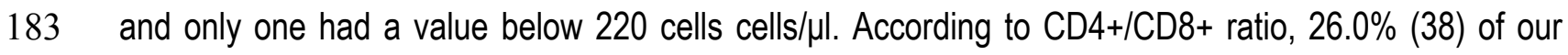
184 population were immunocompromised (ratio<0.5). The majority $(75.3 \%)$ of the 146 study participants had 185 a controlled of viral replication $(<40$ copies $/ \mathrm{mL}$ ) while $12.3 \%(18)$ were experiencing virological failure 186 (>1000 copies $/ \mathrm{mL}$ ) (Table 3).

\section{Correlation between immuno-virological parameters and nutritional profile}

We found a negatively weak correlation between CD4+ T cells count and total protein concentration $(r=-$ $0.18 ; p=0.03)$ as shown on figure $1 . \mathrm{CD} 8+$ showed a negatively weak correlation with glucose concentration ( $r=-0.24 ; p=0.003)$ and a positively weak correlation with total protein concentration $(r=0.18$; $p=0.031$ ) as shown in figure 2 and figure 3 respectively. Bivariate analysis of viremia with each nutritional parameter are presented in table 4. Viral load showed a negatively weak correlation between with total cholesterol $(r=-0.28, p=0.0007)$, and a positively weak correlation with total protein $(r=0.28, p=0.0006)$ and with triglycerides $(r=0.20, P=0.018)$, as shown figure 4 , figure 5 and figure 6 respectively. After multivariate analysis including BMI, WLP, NRI, total cholesterol, total protein, triglycerides and albumin, three parameters (total cholesterol, total protein and triglycerides) were found to be independently correlated to viral load, as shown on table 5.

\section{Discussion}

201 In the present study whose aim was to determine the correlation between the immuno-virological 202 response and the nutritional profile of patients on ART within the Cameroonian context, the majority of 203 our study participants were predominantly female (76.0\%). This is similar to data in the same country 204 reported by Ndawinz, et al. (68.1\%), the Cameroon Population-based HIV Impact Assessment in 2017 205 (CAMPHIA) and the National Health and Demographic Survey (HDS) data in $2019[17,18,27,28]$, thus confirming the biological vulnerability of women to HIV infection. The median age $[I Q R]$ of the study participants was 42 [33-51] years. This could be explained by the fact that the vast majority of infected

208 individuals in Cameroon lie within this age interval, as found by Billong et al., in 2012 and previous survey data in Cameroon[17,18,20]. 
According to Body Mass Index (BMI), about 2 out of 5 participants were undernourished, suggesting that poor nutritional status is a major concern among patients receiving ART. Similar findings were recently reported in Ethiopia, which underscores the fact that beyond adherence to an effective ART-regimen,

213 malnutrition remains a threat that deserves special considerations [29]. The fact that weight loss was

214 found in $40 \%$ of our participants confirms the uniqueness of HIV infection in inducing a progressive decline

215 of body weight $[30,31]$. Our data also revealed that about three quarters of participants lived on an average

216 daily ration that range between $\$ 2$ and $\$ 10$ per day, and only half of participants could afford 2 meals per

217 day, which in turn justifies the profile of WLP and highlights the financial constraint HIV could have on

218 food insecurity among Cameroonian families [32].

219 About $79 \%$ of our participants had a normal level of total protein $(66-88 \mathrm{~g} / \mathrm{L})$, as previously reported in a similar African setting [29]. This profile also suggests upregulation of antibody production in an attempt to compensate ongoing immunodeficiency [33]. About half of our participants had a normal immunity (547 median CD4+ T cells), similar to previous reports in Cameroon [34,35]. The negatively weak correlation between CD4+ T cells and total protein was previously reported by Lyer et al. in a population of black Americans, with a similar positive trend observed with CD8 cells [36,37]. These states of immunity (CD4+ and CD8+) reflect the good response to ART [38]. The negatively weak correlation between CD8+ T cells and glucose suggests that HIV-associated alterations in CD8+ T-cell function also impair glycolysis, thus hindering glucose metabolism in HIV infection [39].

About $75 \%$ and $88 \%$ of participants achieved viral undetectability $(<40 \%)$ and viral suppression $(<1000$ copies $/ \mathrm{mL}$ ) respectively. Similar to previous reports [34], these findings suggests that achieving the third 230 pillar of the 90-90-90 is possible and could be reinforced with adequate nutrition in the frame of an effective 231 ART [40].

232 Following multivariate analysis, total protein, total cholesterol and triglycerides were independent factors 233 correlated to viral load. Of note, HIV infection could trigger a loss in total protein [41], which in turn lead 234 to an increasing risk of proteinemia in the frame of an increasing viral load and CD4 count $<200 / \mathrm{mm}^{3}$ [36]. 235 Increased viral load is also known to impair cholesterol level [42], suggesting interaction between HIV and 236 cholesterol leading to metabolic abnormalities (lipodystrophy, dyslipidemia, diabetes mellitus, and insulin 237 resistance) prone by HIV itself and/or antiretroviral agents [43]. With a high correlation, total cholesterol 238 thus appear to play an important role in HIV life cycle owing to lipid functions in viral entry, uncoating, 239 replication, protein synthesis, assembly, budding and infectivity [44].

240 Study limitations: Our study covered only a single region of Cameroon, making generalizability difficult at 241 country level. It would have been insightful to assess pro and anti-inflammatory markers with regards to 242 ART response and variability of the nutritional status. In addition, understanding the impact of immune 
Nutrition and HIV

activation induced by ART would have help in delineating covariates of nutritional parameters, immune response and accelerated ageing among patients receiving ART in our context.

\section{Conclusion}

247 In this resources limited sitting, with patients having about five years of ART-experience, half are

248 immunocompromised while the majority have achieved good virological response. Interestingly, one out 249 of eight patients might be experiencing malnutrition. Specifically, increasing CD4 may favour hypo250 proteinemia while increasing viral load may prone hyper-proteinemia and hypo-cholesterolemia. Further 251 studies are needed in resources limited sitting with high burden of HIV-infection.

\section{Supporting Information}

254 SI File (questionnaire)

\section{Figures and legends}

256 Figure 1: Correlation between LTCD4+ and Total protein

257 Figure 2: Correlation between LTCD8+ and Total Protein

258 Figure 3: Correlation between LTCD8+ and Glucose

259 Figure 4: Correlation between viral load and total cholesterol

260 Figure 5: Correlation between viral load and total protein

261 Figure 6: Correlation between viral load and triglycerides

\section{Tables and legends}

\section{Table 1: Socio-demographic data}

\begin{tabular}{lll}
\hline Variables & Effective & Percentage (\%) \\
\hline Health Center & 129 & 88.4 \\
BRH & 17 & 11.6 \\
NCHC & & \\
Gender & 35 & 23.9 \\
Male & 35
\end{tabular}


Nutrition and HIV

\begin{tabular}{lll}
\hline Female & 111 & 76.0 \\
$\begin{array}{l}\text { Economic status per day (Francs CFA) } \\
<500\end{array}$ & 8 & 5.5 \\
$500-1000$ & 27 & 18.5 \\
$1000-5000$ & 107 & 73.3 \\
$>5000$ & 4 & 3.7 \\
Average of meals per day & & \\
1 meal & 12 & 8.2 \\
2 meals & 72 & 49.3 \\
3 meals & 54 & 36.9 \\
4 meals & 8 & 5.5
\end{tabular}

Legend: BRH: Bertoua regional hospital; NCHC: Nkolbikon Catholic Health Center

267 Table 2: Anthropometric and biochemical parameters

\begin{tabular}{lll}
\hline Variables & Effective & Percentage (\%) \\
\hline BMI & 42 & 28.7 \\
$\quad$ Overweight: $>25.0$ & 87 & 59.6 \\
$\quad$ Not malnourished: $18.5-25.0$ & 17 & 11.6 \\
$\quad$ Malnourished: $<18.5$ & & \\
NRI & 133 & 91.1 \\
$\quad$ Not malnourished: $>100$ & 5 & 3.4 \\
$\quad$ Mild malnutrition: $97.5-100$ & 8 & 5.5 \\
$\quad$ Moderate malnutrition: $83.5-97.5$ & 0 & 0.0 \\
$\quad$ Severe malnutrition: $<83.5$ & & \\
WL & 89 & 60.9 \\
$\quad$ Normal weight: $0 \%$ & 50 & 34.2 \\
$\quad$ Mild underweight: $-5 \%-0 \%$ & 7 & 5.8 \\
Advanced underweight: $-10 \%-(-5 \%)$ & 0 & 0.0 \\
$\quad$ Severe underweight: $<-10 \%$ & & \\
\hline
\end{tabular}

\section{Biochemical parameters}

Albumin: $37-53 \mathrm{~g} / \mathrm{l}$

Calcium: $86-103 \mathrm{mg} / \mathrm{l}$

High Normal Low High Normal Low

$\begin{array}{llllll}6 & 136 & 4 & 4.1 & 93.2 & 2.7 \\ 13 & 105 & 28 & 8.9 & 71.9 & 19.2\end{array}$


Nutrition and HIV

$\begin{array}{lllllll}\text { Glucose: } 0.7-1.15 \mathrm{~g} / \mathrm{l} & 4 & 111 & 31 & 2.7 & 76.0 & 21.2 \\ \text { Iron: } 0.5-1.6 \mathrm{mg} / \mathrm{l} & 37 & 90 & 19 & 25.3 & 61.6 & 13.0 \\ \text { Magnesium: } 13-21 \mathrm{mg} / \mathrm{l} & 120 & 24 & 2 & 82.2 & 16.4 & 1.4 \\ \text { Total cholesterol: } 1.4-2.0 \mathrm{~g} / \mathrm{l} & 5 & 111 & 30 & 3.4 & 76.0 & 20.5 \\ \text { Total protein: } 63-83 \mathrm{~g} / \mathrm{l} & 29 & 115 & 2 & 19.9 & 78.7 & 1.4 \\ \text { Triglycerides: } 0.0-2.0 \mathrm{~g} / \mathrm{l} & 9 & 137 & 0 & 6.2 & 93.8 & 0.0\end{array}$

Legend: BMI: Body Mass Index;

NRI: Nutritional Risk Index;

WLP: Weight Loss Percentage

Table 3: Immuno-virological results

$\mathrm{N}$

$\%$

CD4 cell (cells/ul)

Below 200

Between 200-349

4

2.7

Between 350-499

25

17.1

Above 500

81

24.6

55.5

CD8 cell (cells/ $\mu l)$

Below 220

0.7

Above 220

138

99.3

CD4/CD8

Below 0.5

38

26.0

Between 0.5-2.0

106

72.6

Between 2.0

2

1.4

ART received

$3 T C+T D F+E F V$

$3 T C+T D F+E F V+C o t r i m$

$3 T C+A Z T+N V P$

$3 T C+A B C+E F V$

$3 T C+A Z T+E F V$

4

2.7

Viremia (copies/mL)

Viral control (undetectable)

Low level viremia (40-1000)

Virological failure (>1000)

12.3 
271 Legend: 3TC: Lamivudine; ABC: Abacavir; ART: Antiretroviral Therapy; AZT: Zidovudine; CD: Cluster of Differentiation; EFV: Efavirenz; NVP: Nevirapine; TDF: Tenofovir

274 Table 1: Correlation between immune-virological parameters and nutritional profile

\begin{tabular}{llll}
\hline & CD4 & CD8 & Viremia \\
\hline Albumin & 0.50 & 0.93 & $\mathbf{0 . 0 1 3}$ \\
BMI & 0.80 & 0.78 & 0.05 \\
Calcium & 0.07 & 0.11 & 0.91 \\
Glucose & 0.87 & $\mathbf{0 . 0 0 3}$ & 0.38 \\
Iron & 0.10 & 0.65 & 0.68 \\
Magnesium & 0.31 & 0.34 & 0.76 \\
NRI & 0.45 & 0.66 & $\mathbf{0 . 0 0 0 9}$ \\
Total Cholesterol & 0.89 & 0.08 & $\mathbf{0 . 0 0 0 7}$ \\
Total protein & $\mathbf{0 . 0 3}$ & $\mathbf{0 . 0 3 1}$ & $\mathbf{0 . 0 0 0 6}$ \\
Triglycerides & 0.58 & 0.46 & $\mathbf{0 . 0 1 8}$ \\
WLP & 0.46 & 0.89 & 0.19 \\
\hline
\end{tabular}

Legend: BMI: Body Mass Index;

NRI: Nutritional Risk Index;

WLP: Weight Loss Percentage

276

Table 2: Multivariate analysis with viral load

\begin{tabular}{|c|c|c|c|c|c|}
\hline & Coefficient & Std. Error & t value & $\operatorname{Pr}(>|t|)$ & \\
\hline Intercept & $\begin{array}{l}-0.98 \\
\end{array}$ & 0.95086 & $\begin{array}{l}-1.039 \\
\end{array}$ & 0.30050 & - Adjusted R-squared: 0.2517 \\
\hline Total cholesterol & -0.65 & 0.19432 & -3.356 & 0.00102 ** & - F-statistic: 13.2 on 4 and $141 \mathrm{DF}$ \\
\hline Total protein & 0.28 & 0.01005 & 5.295 & $4.46 \mathrm{e}-07^{* * *}$ & - $p$-value: $3.71 \mathrm{e}-09$ \\
\hline Triglycerides & 0.27 & 0.15345 & 1.770 & 0.07894 & \\
\hline
\end{tabular}

We are grateful to all HIV-clinic attendees at the care unit of the two different Health Facilities selected in the East region of Cameroon (Bertoua Regional Hospital and Nkolbikon Catholic Health Center). We are

282 also thankful to the lab-technicians and clinicians of these sites who contributed in the field for enrolment; 283 and the entire staff of the CIRCB for contributing in the lab-analysis process. 


\section{Author Contributions}

286 Designed the study: AA, RSK, JF, SMS, AN.

287 Collected the data: AA, ADN, ENS.

288 Analyzed and Interpreted the data: AA, ACK, MTT, ACC, JFY.

289 Initiated the manuscript: AA, RSK, JF, SMS, AN.

290 Revised the manuscript: ADN, ENS, ACK, MTT, ACC, YJ.

291 Approved the final version: All the authors.

\section{References}

294 [1] Tudela EV, Singh MK, Lagman M, Ly J, Patel N, Venketaraman V. Cytokine Levels in Plasma 295 Samples of Individuals with HIV Infection n.d.:5.

296 [2] Labban L. The Implications of HIVIAIDS on the Nutritional Status and the MNT for Its Patients 297 2016:7.

298 [3] Nahlen BL, Chu SY, Nwanyanwu OC, Berkelman RL, Martinez SA, Rullan JV. HIV wasting 299 syndrome in the United States. AIDS 1993;7.

300 [4] Kotler DP, Tierney AR, Wang J, Pierson RN. Magnitude of body-cell-mass depletion and the 301 timing of death from wasting in AIDS. Am J Clin Nutr 1989;50:444-7. 302 https://doi.org/10.1093/ajcn/50.3.444.

303 [5] Matthias Rath, Alexandra NIEDZWIECKI. Malnutrition: The Leading Cause of Immune Deficiency 304 Diseases Worldwide. Dr. Rath Research Institute; 2005.

305 [6] Gredel S. Nutrition and immunity in man. Brussels: ILSI Europe; 2011.

306 [7] Sicotte M, Langlois ÉV, Aho J, Ziegler D, Zunzunegui MV. Association between nutritional status 307 and the immune response in HIV + patients under HAART: protocol for a systematic review. Syst Rev 308 2014;3:9. https://doi.org/10.1186/2046-4053-3-9.

309 [8] Piwoz E, Greble E. HIVIAIDS and Nutrition: A Review of the Literature and Recommendations 310 for Nutritional Care and Support in Sub - Saharan Africa. SARA Support Anal Res Afr Proj 2000.

311 [9] Enwonwu CO. Complex interactions between malnutrition, infection and immunity: relevance to 312 HIVIAIDS infection. Biomed Res n.d.:9.

313 [10] Williams BG, Gouws E. Ending AIDS in South Africa: How long will it take? How much will it cost? 314 n.d.:8.

315 [11] Fields-Gardner C. Position of the American Dietetic Association: Nutrition Intervention and 316 Human Immunodeficiency Virus Infection. J Am Diet Assoc 2010;110:1105-19. 317 https://doi.org/10.1016/j.jada.2010.05.020. 
318 [12] Simon Collins, Jane Shepherd, Roy Trevelion, John Walter, Jonathan Bassett. ART in pictures:

319 HIV treatment explained. i-base; 2017.

320 [13] World Health Organization. Estimated antiretroviral therapy coverage among people living with

321 HIV (\%). World Health Organization 2018. https://www.who.int/data/gho/data/indicators/indicatorJanuary 30, 2020).

324 [14] WHO Technical Consultation on Nutrient Requirements for People Living with HIVIAIDS. Nutrient requirements for people living with HIVIAIDS report of a technical consultation, World Health Organization, Geneva, 13-15 May 2003. Geneva: World Health Organization; 2003.

[15] Moore R, Friedl K, Kramer T, Martinez-Lopez L, Hoyt R. Changes in Soldier Nutritional Status and Immune Function During the Ranger Training Course 1992:175.

329 [16] National Institute of Statistics. Demographic and Health survey and Multiple Indicators Cluster

330 Survey. Demographic and Health survey and Multiple Indicators Cluster Survey DHS-MICS 2011:

331 Preliminary Report. Cameroon: National Institute of Statistics. Demographic and Health survey and

332 Multiple Indicators Cluster Survey; 2012.

333 [17] Anne Cecile Zoung Kanyi Bissek. Cameroon population-based HIV impact assessment resulst:

334 A drop that count. Yaoundé, Cameroon: CAMPHIA; 2018.

335 [18] Institut National de la Statistique (INS), et ICF. 2019. Enquête Démographique et de Santé du 336 Cameroun 2018. Indicateurs Clés. Yaoundé, Cameroun, et Rockville, Maryland, USA : INS et ICF.

337 [19] Observateur national de la santé publique National. Health Analytical Profile 2016 Cameroon 3382016.

339 [20] Billong S, Fokam J, Bissek A, Onambele G, Guegang C, Temgoua Saounde E, et al. Nutritional 340 Profile in Households of HIV-Infected Patients receiving Antiretroviral Therapy in the 10 Regions of 341 Cameroon: Toward the Implementation of Specific Food Interventional Programs. Rev Médecine Pharm 342 2012; Volume 2:185-95.

343 [21] Comité National de lutte contre le VIH/SIDA. REVUE DES ACTIVITES DE LUTTE CONTRE LE 344 VIH/SIDA AU PREMIER SEMESTRE 2017. Ministère de la santé; 2017.

345 [22] World Health Organisation. Laboratory Guidelines for enumerating CD4 T Lymphocytes in the 346 context of HIVIAIDS. New Dehli: 2007.

347 [23] Gize A, Mathewos B, Moges B, Workineh M, Gedefaw L. Establishment of Normal Reference 348 Intervals for CD3 +, CD4 +, CD8 +, and CD4 + to CD8 + Ratio of T Lymphocytes in HIV Negative Adults 349 from University of Gondar Hospital, North West Ethiopia. AIDS Res Treat 2014;2014:1-7. 350 https://doi.org/10.1155/2014/267450.

351 [24] Loh KW, Vriens MR, Gerritsen A, Steenhagen E, Ong TA, Moy FM, et al. Unintentional weight 
loss is the most important indicator of malnutrition among surgical cancer patients 2012;70:5.

[25] Aziz EF, Javed F, Pratap B, Musat D, Nader A, Pulimi S, et al. Malnutrition as Assessed by Nutritional Risk Index is Associated with Worse Outcome in Patients Admitted with Acute Decompensated Heart

Failure:

An ACAP-HF

Data

Analysis. Heart Int

2011;6:hi.2011.e2. https://doi.org/10.4081/hi.2011.e2.

[26] Mazumder S. Determination of Weight loss Percentage After Bleaching of Cellulose Fabrics with Various Bleaching Agents n.d.:7.

[27] Ndawinz JDA, Chaix B, Koulla-Shiro S, Delaporte E, Okouda B, Abanda A, et al. Factors associated with late antiretroviral therapy initiation in Cameroon: a representative multilevel analysis. J Antimicrob Chemother 2013;68:1388-99. https://doi.org/10.1093/jac/dkt011.

[28] Awuba J, Macassa G. HIVIAIDS in Cameroon: Rising gender issues in policy-making matters. Afr J Health Sci 2008;14. https://doi.org/10.4314/ajhs.v14i3.30857.

364 [29] Feleke DG, Yemanebrhane N, Gebretsadik D. Nutritional Status and CD4 Cell Counts in HIVIAIDS Patients under Highly Active Antiretroviral Therapy in Addis Ababa, Ethiopia. J AIDS Clin Res 2017;08. https://doi.org/10.4172/2155-6113.1000688.

[30] Grinspoon S, Mulligan K, Department of Health and Human Services Working Group on the Prevention and Treatment of Wasting and Weight Loss. Weight Loss and Wasting in Patients Infected with Human Immunodeficiency Virus. Clin Infect Dis 2003;36:S69-78. https://doi.org/10.1086/367561.

[31] van Griensven J, Zachariah R, Mugabo J, Reid T. Weight loss after the first year of stavudinecontaining antiretroviral therapy and its association with lipoatrophy, virological failure, adherence and CD4 counts at primary health care level in Kigali, Rwanda. Trans R Soc Trop Med Hyg 2010;104:751-7. https://doi.org/10.1016/j.trstmh.2010.08.016.

[32] Tanyi PL, Pelser A, Okeibunor J. HIVIAIDS and older adults in Cameroon: Emerging issues and implications for caregiving and policy-making. SAHARA-J J Soc Asp HIVAIDS 2018;15:7-19. https://doi.org/10.1080/17290376.2018.1433059.

[33] Oguntibeju O, van den Heever, Schalkwyk V. The interrelationship between nutrition and the immune system in HIV infection: A review. ANSInet 2007:10(24): 4327-4338.

379 [34] Fokam J, Billong SC, Jogue F, Moyo Tetang Ndiang S, Nga Motaze AC, Paul KN, et al. Immuno380 virological response and associated factors amongst HIV-1 vertically infected adolescents in Yaoundé381 Cameroon. PLOS ONE 2017;12:e0187566. https://doi.org/10.1371/journal.pone.0187566.

382 [35] MC OA, Mouladje M, Ikomey Mondinde G, Adiogo D, Esiène A, Ndumbe P, et al. Valeurs des 383 lymphocytes tcd4 et cd8 chez lez donneurs de sang à yaoundé, Cameroun. Health Sci Dis 2011;12 (4):6. 384 [36] Ramezani A, Mohraz M, Banifazl M, Jam S, Gachkar L, Yaghmaie F, et al. Frequency and associated factors of proteinuria in Iranian HIV-positive patients. Int J Infect Dis 2008;12:490-4. 
https://doi.org/10.1016/j.jijid.2008.01.009.

387 [37] lyer SS, Chatraw JH, Tan WG, Wherry EJ, Becker TC, Ahmed R, et al. Protein Energy 388 Malnutrition Impairs Homeostatic Proliferation of Memory CD8 T Cells. J Immunol 2012;188:77-84. 389 https://doi.org/10.4049/jimmunol.1004027.

390 [38] Sukumar M, Liu J, Ji Y, Subramanian M, Crompton JG, Yu Z, et al. Inhibiting glycolytic metabolism 391 enhances CD8+ T cell memory and antitumor function. J Clin Invest 2013;123:4479-88. 392 https://doi.org/10.1172/JCl69589.

393 [39] Willig AL, Overton ET. Metabolic Complications and Glucose Metabolism in HIV Infection: A 394 Review of the Evidence. Curr HIVIAIDS Rep 2016;13:289-96. https://doi.org/10.1007/s11904-016-0330395 z.

396 [40] Joint United Nations Programme on HIVIAIDS. Blood safety and HIV: UNAIDS Technical Update 3971997.

398 [41] Patil R, Raghuwanshi U. Serum protein, albumin, globulin levels, and A/G ratio in HIV positive 399 patients 2009;2:5.

400 [42] Steenkamp L, Dannhauser A, Walsh D, Joubert G, Veldman F, Van der Walt E, et al. Nutritional, 401 immune, micronutrient and health status of HIV-infected children in care centres in Mangaung. South Afr 402 J Clin Nutr 2009;22:131-6. https://doi.org/10.1080/16070658.2009.11734234.

403 [43] Marzel A, Kouyos RD, Reinschmidt S, Balzer K, Garon F, Spitaleri M, et al. Dietary Patterns and 404 Physical Activity Correlate With Total Cholesterol Independently of Lipid-Lowering Drugs and 405 Antiretroviral Therapy in Aging People Living With Human Immunodeficiency Virus. Open Forum Infect 406 Dis 2018;5. https://doi.org/10.1093/ofid/ofy067.

407 [44] Adal M, Howe R, Kassa D, Aseffa A, Petros B. Associations of gender and serum total cholesterol 408 with CD4+ T cell count and HIV RNA load in antiretroviral-naïve individuals in Addis Ababa. BMC Public 409 Health 2018;18:943. https://doi.org/10.1186/s12889-018-5852-4. 


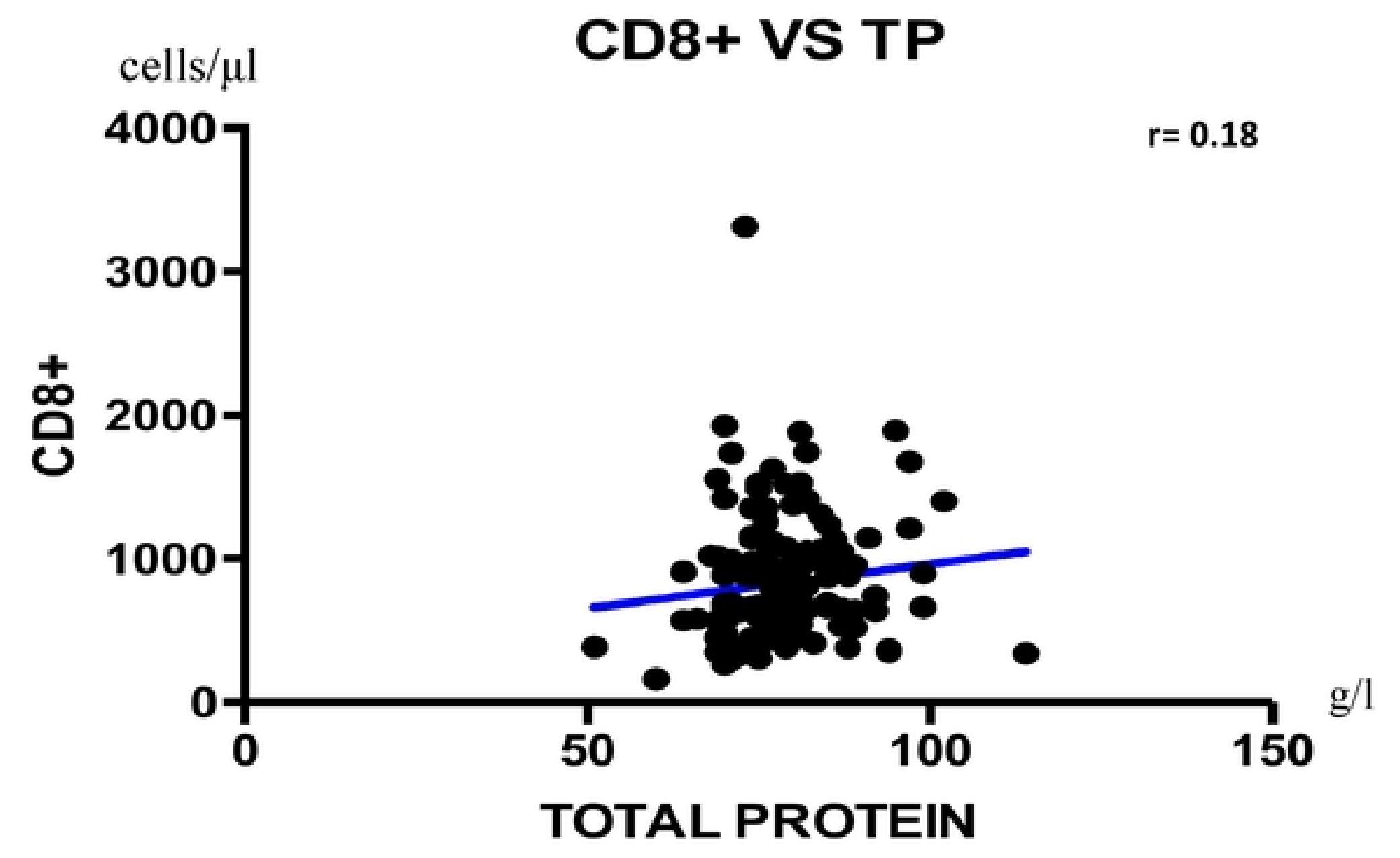

Figure 


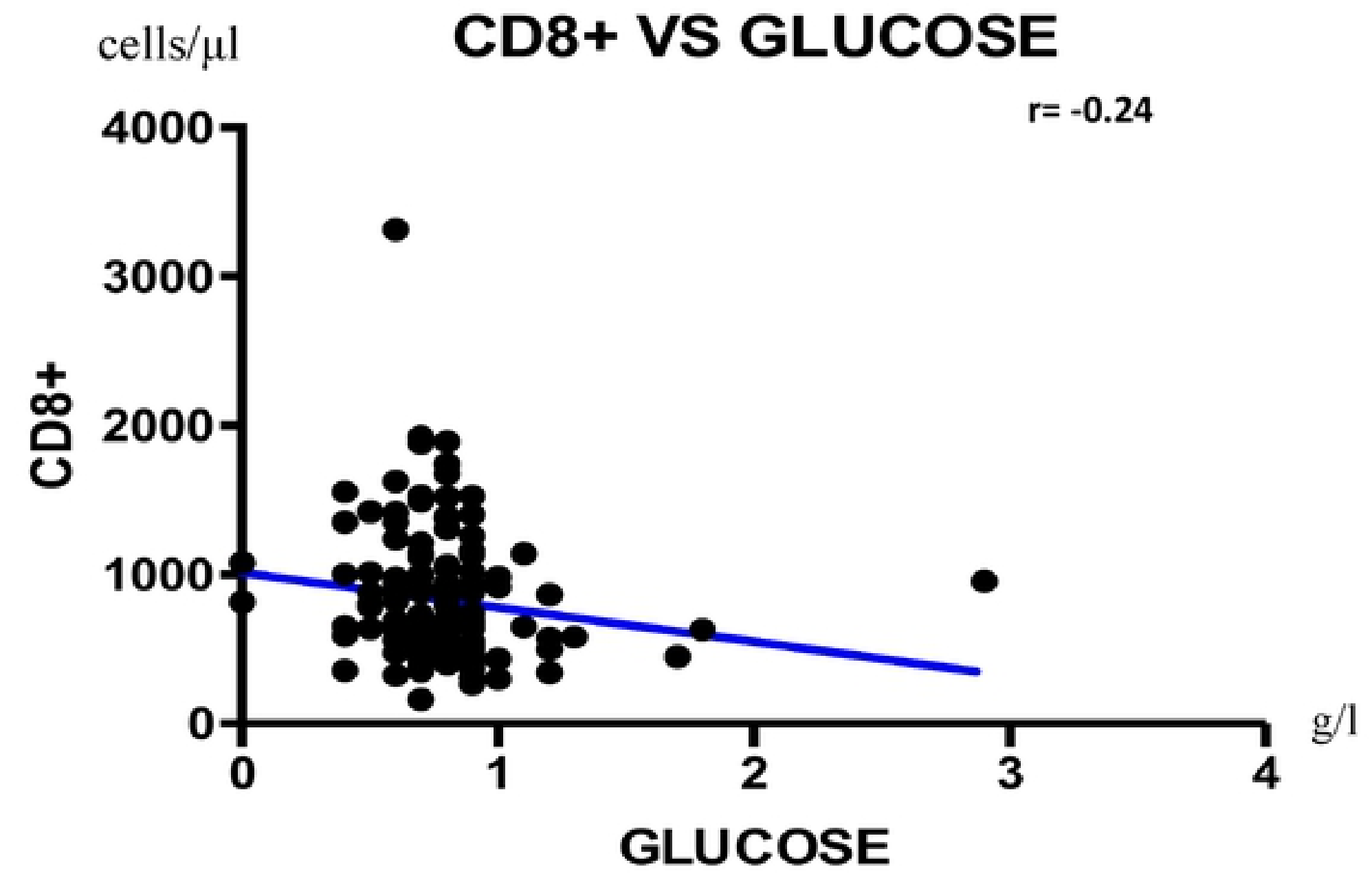

Figure 


\section{VL VS TC}

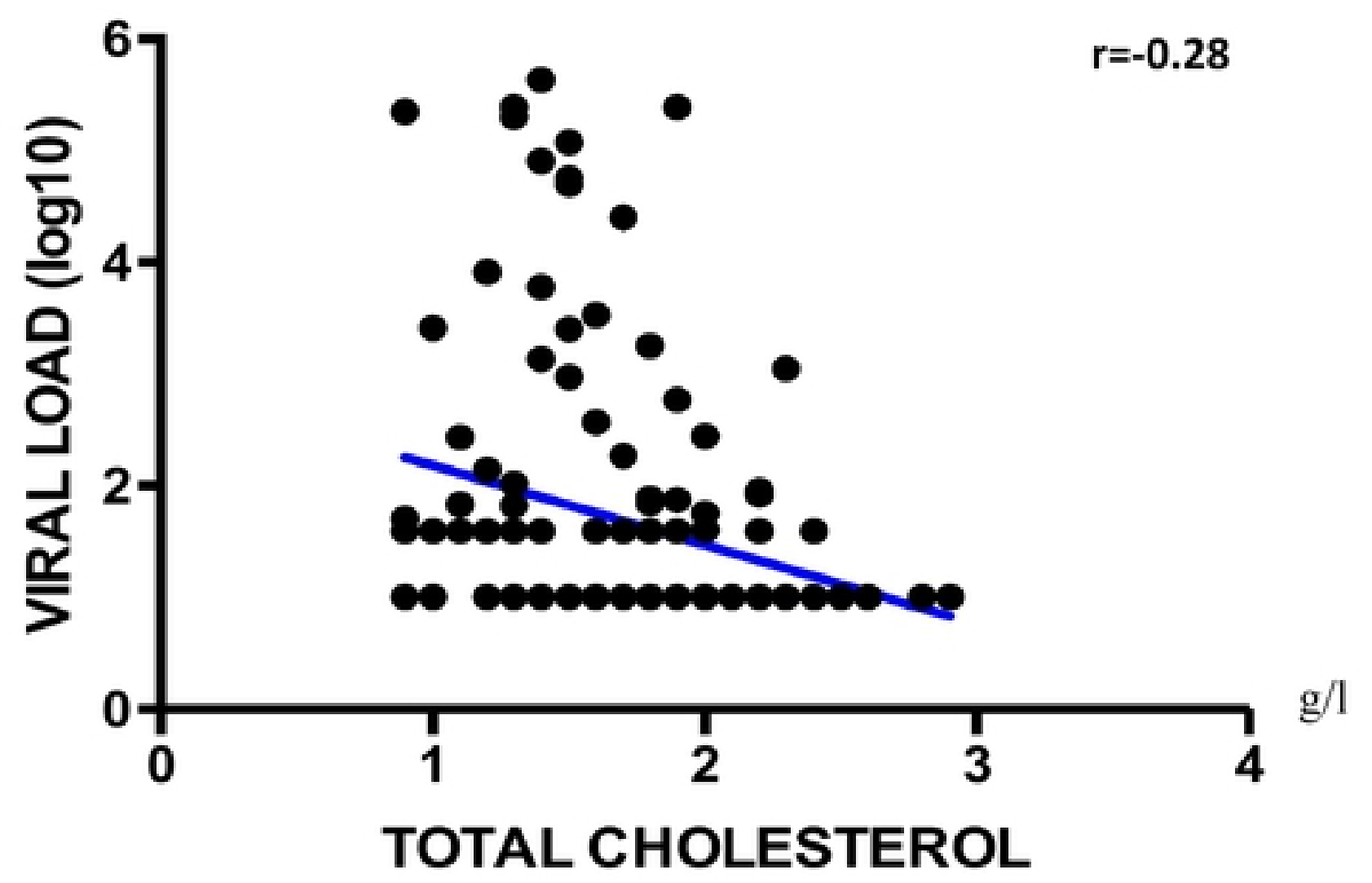

Figure 


\section{VL VS TP}

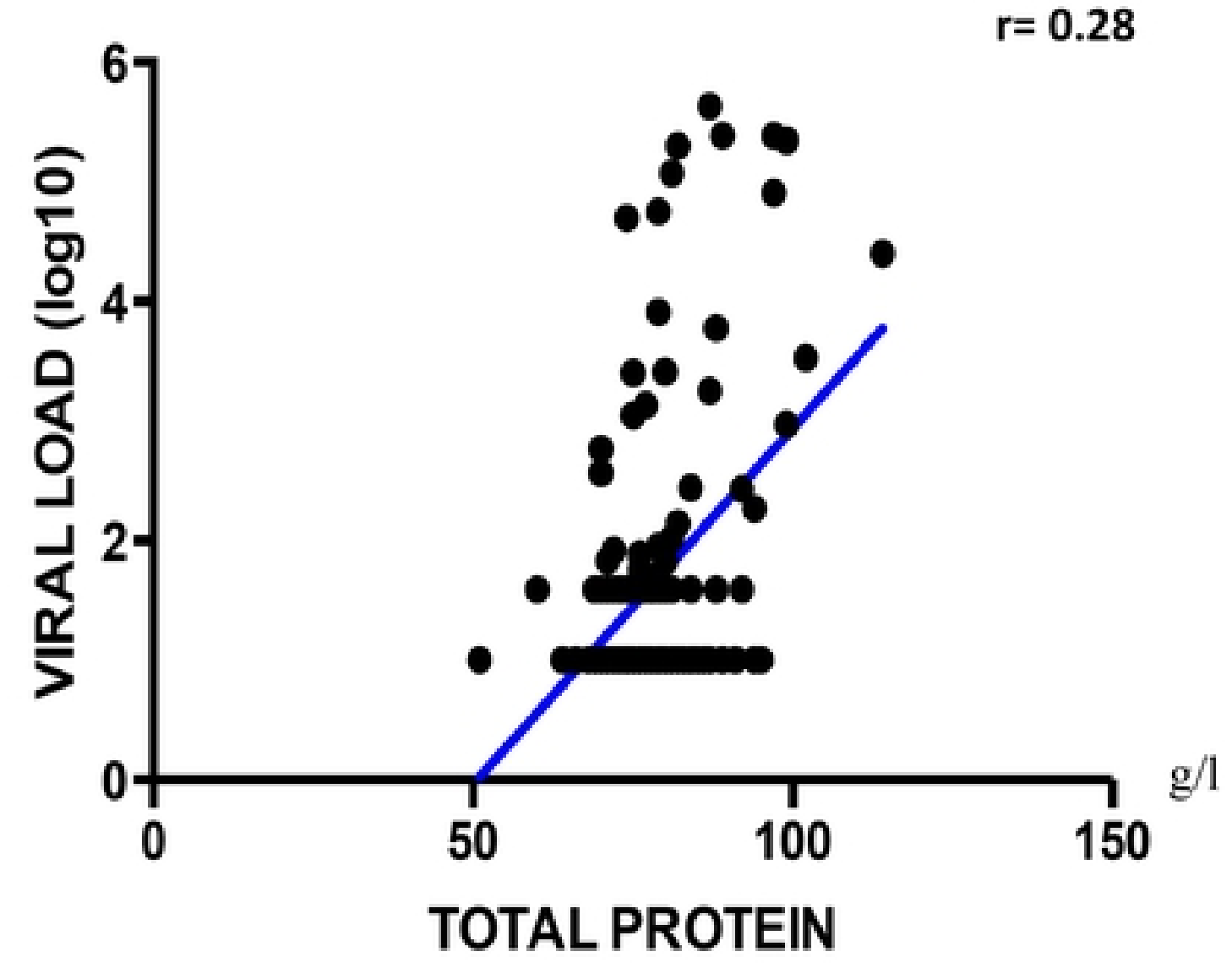

Figure 


\section{VL VS TRIGLY}

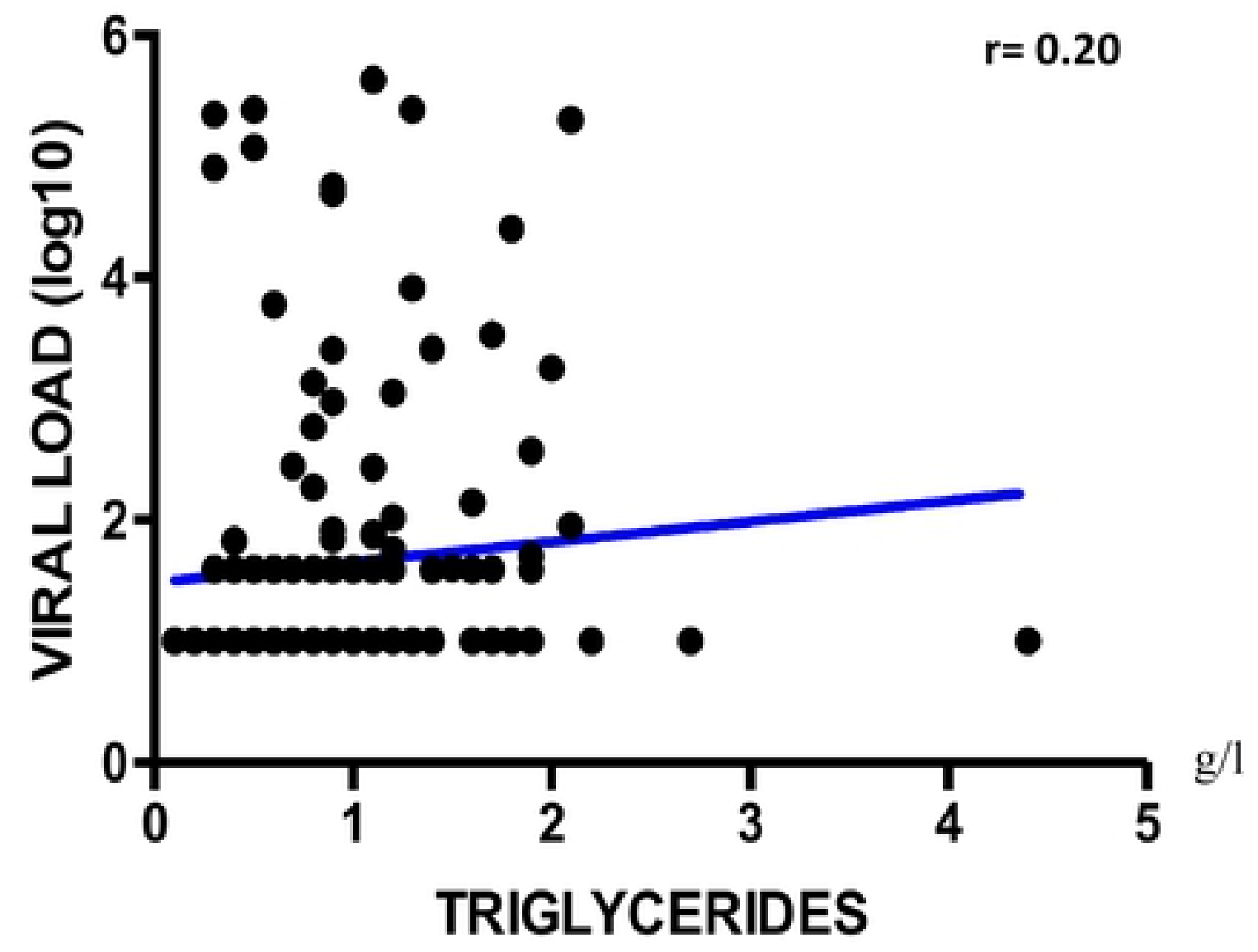

Figure 


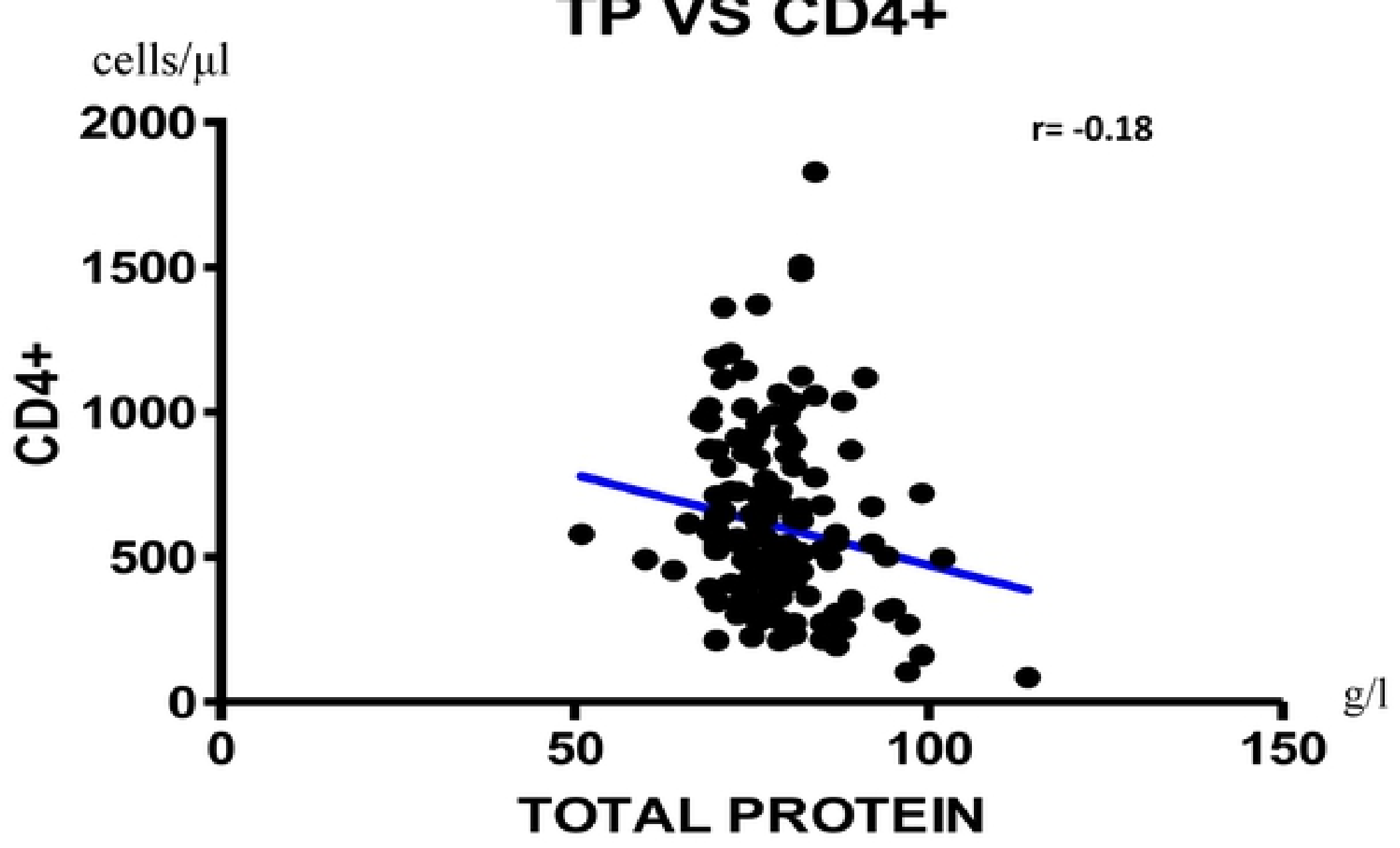

Figure 


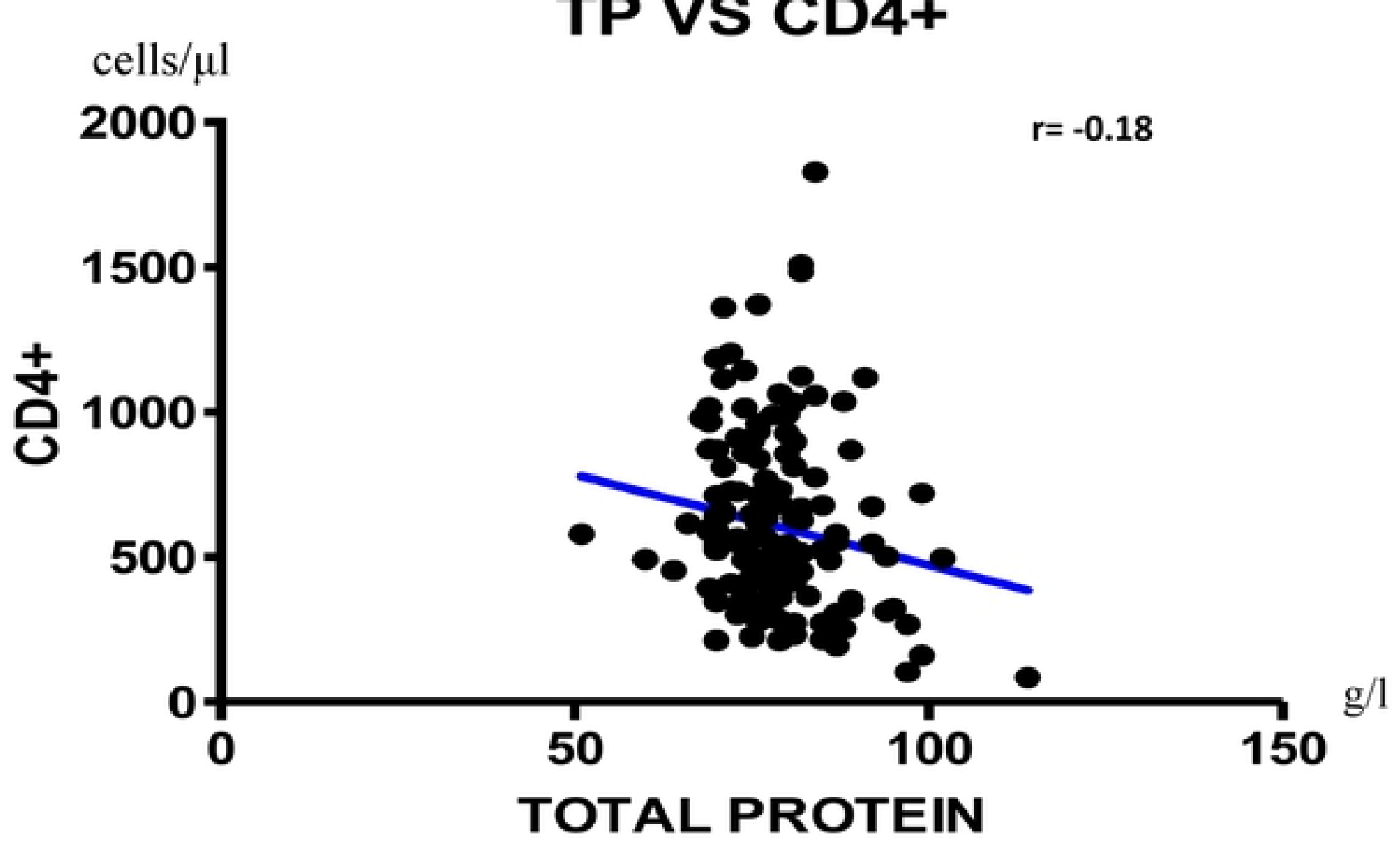

Figure 